\title{
EFFECTIVENESS OF 2-D VIEWS FOR 6-D ROBOTICS SIMULATION MANEUVERS
}

\author{
Teryn M. Bray, Randa L. Shehab, Robert E. Schlegel, Aylin Civan, and Daniel E. Walker \\ School of Industrial Engineering, University of Oklahoma \\ Norman, OK 73019
}

\begin{abstract}
This study examined operator performance on 6-D robotics simulation maneuvers using a single 2-D view of the robot arm. The BORIS simulator, developed by NASA to support Generic Robotics Training, was used to present four "fly-to" tasks with views representing combinations of good and poor control-display movement compatibility and perceptual quality. Translational $(\mathrm{x}, \mathrm{y}, \mathrm{z})$ and rotational (pitch, yaw, roll) accuracies were used to assess performance. A significant interaction between movement compatibility and perceptual quality was found for both the overall translational and y accuracy measures ( $p=0.0289$ and $p=0.0155$, respectively). This interaction appeared to indicate that performance was hindered only if both factors were poor. Perceptual quality significantly affected the $\mathrm{z}$ accuracy and pitch accuracy measures $(p=$ 0.0461 and $p=0.0429$, respectively) with the views identified as poor perceptual quality actually yielding better performance. These results suggest that performance on a 6-D robotics maneuver is not necessarily hindered by using only a 2-D view. If a single view cannot simultaneously provide both good movement compatibility and good perceptual quality, the results suggest that either one is sufficient with respect to overall translational accuracy.
\end{abstract}

\section{BACKGROUND}

The National Aeronautics and Space Administration (NASA) utilizes robots in space to accomplish a variety of tasks including manipulating payloads, transporting EVA astronauts from one location to another, and repairing space equipment. Most of these activities support the construction of the International Space Station (ISS). NASA utilizes generic robotics training (GRT) to aid astronauts in acquiring the basic skills to operate the robotic arms on the ISS and the space shuttles. The GRT program is designed to teach basic knowledge of robotics terminology, robotics technologies, kinematics, system capabilities and constraints, and operational rules, procedures, and strategies. The Basic Operational Robotics Instructional System (BORIS) is the simulator used for training within GRT and provides trainees with computer-based experience in maneuvering a simulated generic robot arm. Through BORIS, astronauts develop a foundation of robotic skills that prepare them to operate the ISS and space shuttle remote manipulator systems (RMS; Todd, 2000).

The BORIS virtual environment is a rectangular room that contains the robot arm and a table for practicing payload manipulations. The robot arm implemented in BORIS has six degrees of freedom: shoulder pitch, shoulder yaw, elbow pitch, wrist pitch, wrist yaw, and wrist roll. The total length of the extended arm is 14 meters with two $6 \mathrm{~m}$ booms (connectors between joints), a $1 \mathrm{~m}$ wrist roll joint, and a $1 \mathrm{~m}$ end effector (used to grasp objects). Within the room, a number of task views are available through cameras as well as through a window in one of the room walls. Cameras are located at each corner of the room, at the robot elbow joint, and on the robot end effector. The cameras on the walls have adjustable pan, tilt, and zoom. The window view is located behind the base of the robot arm. While operating BORIS, operators can utilize as many as three simultaneous views on the display monitors.

\section{PROBLEM DEFINITION}

During GRT, astronauts learn to select from the available task views to find the combination of views that best aids task performance. The selection of views is of utmost importance when operating robot arms in space and within BORIS; the views are the primary means for visualizing robot arm maneuvers and positions. The selection of appropriate views becomes increasingly important with regard to the ability to provide good control-response compatibility and good perceptual quality.

Visual-motor compatibility has been shown to have significant effects on teleoperator performance. High 
visual-motor compatibility is achieved when the motion direction of the cursor on a display, as viewed by the operator, matches the motion direction of the control device. Misalignments of the control and display axes that reduced visual-motor compatibility resulted in significantly increased RMS error on a tracking task (Macedo, Kaber, Endsley, Powanusorn, \& Myung, 1998). For this reason, selection of views providing good visual-motor compatibility is very important to successful operation.

Van Erp and Oving (2002) conducted an experiment to investigate control accuracy in three dimensions ( $x, y$, and $\mathrm{z}$ ). They employed two tracking tasks to distinguish the influence of visual effects (mapping differences) and psychomotor effects (axis differences) on accuracy. The authors concluded that the quality of visual information, particularly in the depth dimension, is critical to maximizing performance (Van Erp \& Oving, 2002).

The selection of the task view affects the controldisplay movement compatibility and perceptual quality of the task. Within the context of this study, controldisplay movement compatibility was defined as the physical relationship between movement of the hand controller and the resulting movement of the end effector. Perceptual quality was defined as the ability of the view to provide position and orientation information simultaneously in all dimensions. In general, it is feasible to select a combination of three different task views that enables both good movement compatibility and good perceptual quality. However, can a single view provide sufficient movement compatibility and/or perceptual quality for effective task performance? The goal of this study was to determine the impact of losses of movement compatibility and perceptual quality on robotics operator performance when using a single task view. Furthermore, when only a single view is available, is it more critical to ensure that the view provides good movement compatibility or good perceptual quality?

\section{Participants}

\section{METHOD}

Three male and three female engineering students attending the University of Oklahoma, Norman campus participated in the study. Students ranged in age from 18 to 25 years. None of the participants had prior experience in operating BORIS or any other robot arms. Participants were paid for their involvement in the study.

\section{Experimental Design}

The study used a $2 \times 2$ factorial design with repeated measures. Movement compatibility and perceptual quality were each defined to have a qualitatively good and a qualitatively poor level. The order in which the participants completed the four tasks representing the factorial combinations was balanced to minimize order effects.

Seven measures of operator performance were used in this study. These measures are shown in Table 1. The translational accuracies of the final position of the end effector were calculated as the deviation of the actual coordinates from the intended coordinates (measured in $\mathrm{cm}$ ). These accuracies were evaluated using individual $\mathrm{x}, \mathrm{y}$, and $\mathrm{z}$ deviations as well as an overall Euclidean distance. Rotational accuracies of the final orientation of the end effector were calculated as the deviation of the actual orientation from the intended orientation (measured in degrees). These accuracies were evaluated along each dimension.

Table 1. Performance Measures.

\begin{tabular}{|c|c|}
\hline Translational & Rotational \\
\hline X Accuracy & Pitch Accuracy \\
\hline Y Accuracy & Yaw Accuracy \\
\hline Z Accuracy & Roll Accuracy \\
\hline Overall Accuracy & \\
\hline
\end{tabular}

\section{Experimental Tasks}

The definition of the experimental tasks required specifying position and orientation coordinates as well as task views. First, four fly-to tasks were formulated. These tasks required the operator to move the robot end effector from a starting coordinate position to an ending coordinate position. The tasks were located in various areas of the simulation room but were defined such that all task movements traveled the same Euclidean distance.

The second step in developing the tasks involved the selection of views to be used. The view for each experimental task was selected from the four cameras located in the room corners and the window located behind the arm base. These views were chosen because they provided the views necessary to judge distances across the room. Further refinement of the views was necessary to select the task view that afforded the desired level of movement compatibility and perceptual quality. Since the cameras had adjustable settings, they first needed to be individually oriented for each fly-to task. Two experienced BORIS operators defined the 
movement compatibility and good and poor perceptual quality. The operators determined the optimal camera (pan, tilt, and zoom) settings by watching BORIS automatically perform each fly-to task. The operators rated these "optimal" task views, and the views with extreme ratings were selected as the levels of the independent variables. An example of a task view providing poor perceptual quality is shown in Figure 1. This view represents poor perceptual quality because depth along the line of sight (which represents lateral arm movement) is not easily judged.

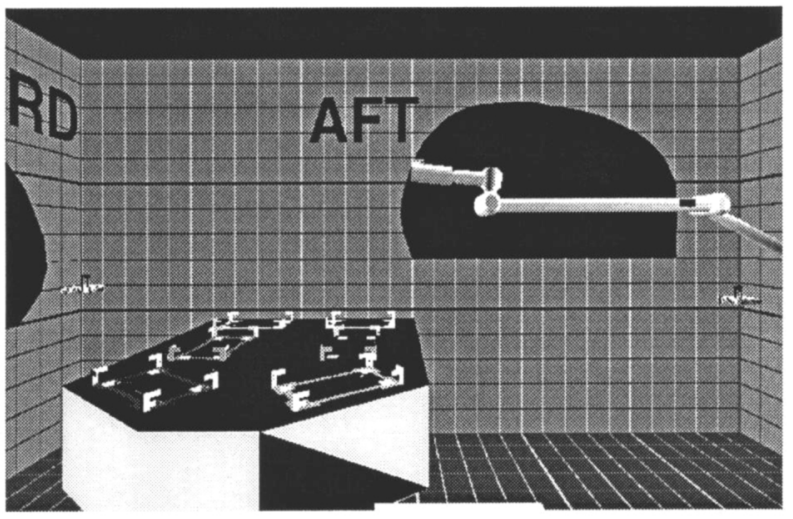

Figure 1. Sample task view showing poor perceptual quality.

Each participant performed four 6-D movement tasks. Each task began with the end effector located at a translational position of $(1200,-100,-500)$ and an orientation of $(0,0,0)$ with respect to the base of the arm. The participant then moved the end effector to the specified set of coordinates for each task. Figure 2 shows a plan view of each task in the X-Y plane. The shaded circle represents the location of the view origin with the dashed line representing the line of sight of the view. The task starting point is shown by the open ellipse, and the direct path to the ending point (filled ellipse) is shown with the arrow.

\section{Procedure}

Participant training and testing spanned five days. Participant training consisted of the first three lessons (two hours each) of the NASA GRT course. The first lesson was completed on day one and covered robotics terminology. The terms and concepts were taught using diagrams, drawings, and 3-D models. The next two lessons were conducted on day two. Lesson 2 introduced the participants to the simulation hardware and allowed them to practice robotics maneuvers. In lesson 3, participants learned about views and camera configurations. The third day of participation involved two hours of hands-on simulation practice in which participants completed standard robotics maneuvers to gain familiarity with the software and hardware.

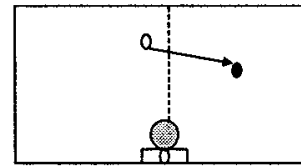

Good MC - Good PQ

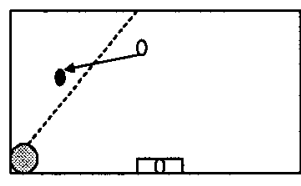

Good MC - Poor PQ

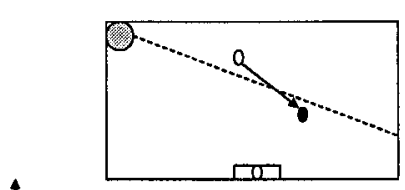

$\begin{array}{ll}\mathbf{x} & \\ & \mathrm{y}\end{array}$

Poor MC · Good PQ

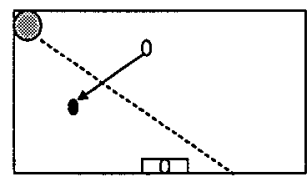

Poor MC - Poor PQ
Figure 2. Plan view of the experimental tasks.

Participants were evaluated on the fifth day of the experiment. For evaluation each participant completed each experimental task once. Participants had one hour in which to complete the four tasks.

\section{RESULTS AND ANALYSES}

The data measured for each fly-to task were evaluated using analysis of variance (ANOVA). Virtually all of the significant effects involved the translational measures of accuracy. Overall translational accuracy was affected by movement compatibility, perceptual quality, and their interaction. Similarly, y accuracy revealed significant main effects and interaction. Perceptual quality was the only significant factor for the measures of $z$ accuracy and pitch accuracy. No significant effects were found for the $\mathrm{x}$ accuracy, yaw accuracy, and roll accuracy measures. A summary of the results is shown in Table 2.

Table 2. Analysis of Variance Summary.

\begin{tabular}{|c|c|c|c|c|}
\hline & \multicolumn{3}{|c|}{ Significance } \\
\hline & & $\begin{array}{c}\text { Movement } \\
\text { Compatibility }\end{array}$ & $\begin{array}{c}\text { Perceptual } \\
\text { Quality }\end{array}$ & Interaction \\
\hline \multirow{4}{*}{ 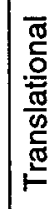 } & Overall Accuracy & $p=.0373$ & $p=.0237$ & $p=.0289$ \\
\hline & $X$ accuracy & & & \\
\hline & Y accuracy & $p=.0196$ & $p=.0082$ & $p=.0155$ \\
\hline & $Z$ accuracy & & $p=.0461$ & \\
\hline \multirow{3}{*}{ 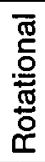 } & Pitch Accuracy & & $p=.0429$ & \\
\hline & Yaw Accuracy & & & \\
\hline & Roll Accuracy & & & \\
\hline
\end{tabular}


Figure 3 shows the significant interaction $(F(1,5)=$ $9.21, p=.0289$ ) of movement compatibility and perceptual quality with respect to overall translational accuracy. This figure indicates that when fly-to tasks are performed with poor movement compatibility, a view that also provides poor perceptual quality results in a much larger deviation from the intended location. However, the influence of poor perceptual quality is minimized when movement compatibility is good. Alternatively, poor movement compatibility has a minimal impact when good perceptual quality is preserved.

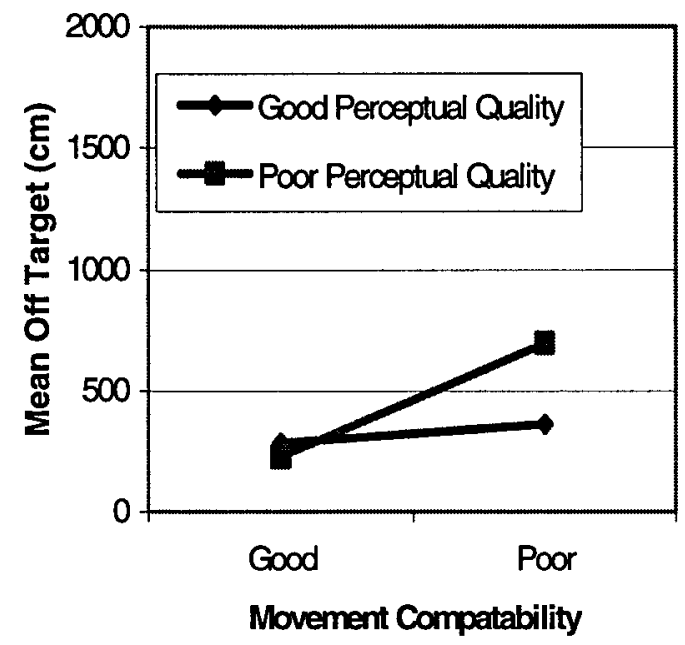

Figure 3. Overall translational accuracy by perceptual quality and movement compatibility.

A significant interaction $(F(1,5)=12.98, p=.0155)$ between movement compatibility and perceptual quality was also found for y accuracy (Figure 4). A similar pattern was observed in these data as was found in the overall translational accuracy. However, poor movement compatibility had a slightly larger negative effect on performance regardless of the perceptual quality afforded by the view. This effect was much stronger when poor movement compatibility was coupled with poor perceptual quality.

Perceptual quality was the only effect that significantly influenced translational accuracy in the $\mathrm{z}$ direction $(F(1,5)=6.95, p=.0461)$. Figure 5 illustrates this main effect. Unexpectedly, the view considered to provide poor perceptual quality resulted in more accurate performance. This condition was also accompanied by a smaller standard deviation (s). A similar pattern of performance was found for the effect of perceptual quality on pitch accuracy $(F(1,5)=7.28, p$ $=.0429$ ).

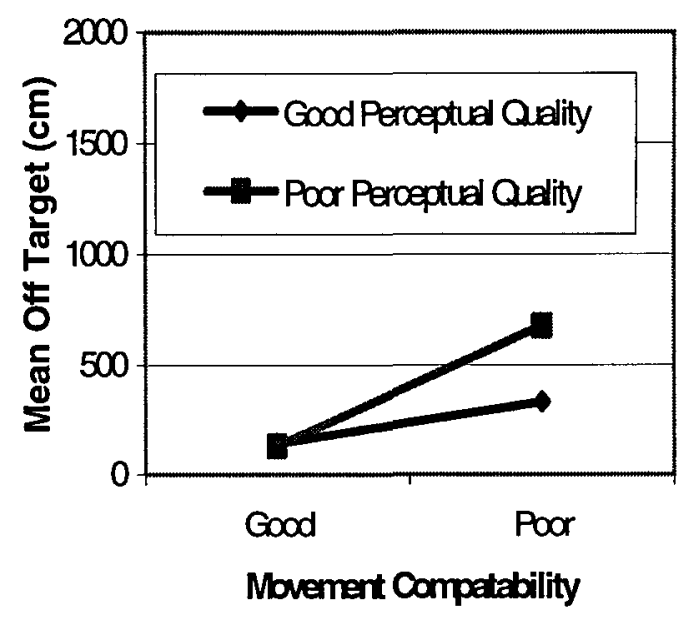

Figure 4. Y accuracy by perceptual quality and movement compatibility.

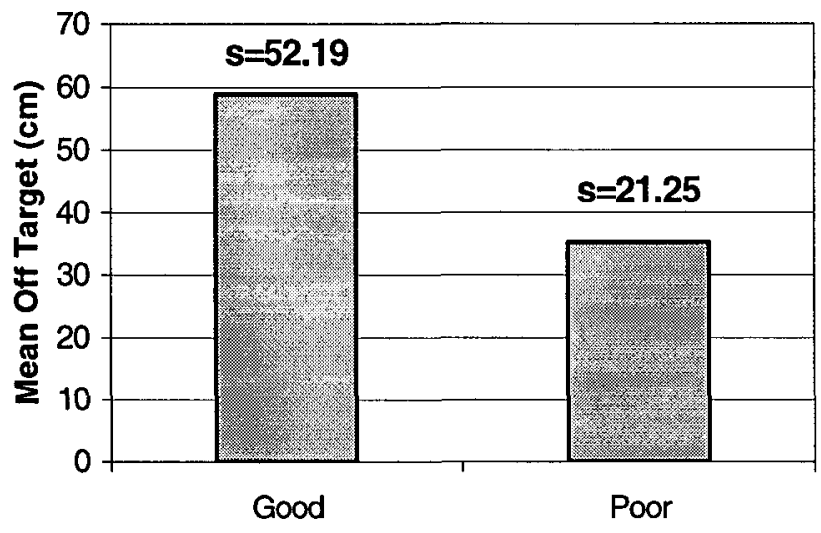

Figure 5. $\mathrm{Z}$ accuracy by perceptual quality.

\section{CONCLUSIONS}

The significant interactions that were identified for the overall translational accuracy and y accuracy measures indicated that neither movement compatibility nor perceptual quality could individually account for poor participant performance. Performance was negatively impacted only when both factors were present at the "poor" level. As long as at least one of the two factors was present at its "good" level, participant performance was not impacted.

The $\mathrm{z}$ accuracy and pitch accuracy measures yielded results that were contrary to expectations. The task views that were defined to be of poor perceptual quality yielded better performance than those views defined to be of good perceptual quality. These results are likely due tonthe fixededration of the alternate task views 
provided within BORIS. The cameras and window used were primarily orthogonal to the $\mathrm{z}$ direction, with only moderate adjustments made to camera tilt. This made it difficult to develop a task with distinctly different levels of good and poor perceptual quality in the $\mathrm{z}$ direction. In addition, the line of sight for the particular task views used in this study was generally aligned with the $y$ direction of movement, making it easier to perceive rotation about the y axis (i.e., pitch) and difficult to create true conditions of poor perceptual quality.

The research question addressed in this study was whether a single task view can provide adequate movement compatibility and perceptual quality for effective and efficient completion of a 6-D robotics task and whether movement compatibility or perceptual quality was more important in selecting the task view. In general, as long as the 2-D view does not have poor movement compatibility AND poor perceptual quality, then the use of a 2-D view will not be detrimental to performance. However, if a single view cannot provide both good movement compatibility and good perceptual quality, the results suggest that either one is sufficient with respect to overall translational accuracy.

\section{FUTURE RESEARCH}

The performance measures analyzed in this study were oriented about a Cartesian coordinate system. However, the views used during the tasks were not oriented along any of these standard axes (except for the window view); they were each individually oriented into 3-D space. This approach resulted in different levels of orthogonality between the view line of sight and the direction of task movement for each task. Thus, performance measures defined along the standard $x y z$ coordinate frame may not reflect true differences.
It is suggested that reanalysis of the data is appropriate such that performance on each task is quantified using a coordinate system aligned with the view line of sight. This will allow investigation of errors in depth perception and along lateral axes.

A second issue to be addressed in future research is the need to operationalize the definitions of movement compatibility and perceptual quality as they pertain to the BORIS environment. The levels of these factors were defined using expert BORIS operators who subjectively evaluated each view for these traits. More precise definitions of these traits would eliminate the subjective evaluation of views and may eliminate the perceptual quality reversal seen in the pitch and $\mathrm{z}$ accuracy data.

\section{ACKNOWLEDGEMENTS}

The work reported here was funded by the U.S. National Aeronautics and Space Administration under NASA Grant NAG 9-1280. The authors would like to thank Michael Buratti and Tamy Fry for their contributions to the design and completion of the study.

\section{REFERENCES}

Macedo, J.A., Kaber, D.B., Endsley, M.R., Powanusorn, P., \& Myung, S. (1998). The effect of automated compensation for incongruent axes on teleoperator performance. Human Factors, 40, 542-553.

Todd, B.K. (2000). Basic Operational Robotics Instructional System, BORIS specifications preliminary.

Van Erp, J.B.F, \& Oving, A.B. (2002). Control performance with three translational degrees of freedom. Human Factors, 44, 144-156. 